\title{
Sarcoma de Kaposi na Odontologia: Um levantamento epidemiológico no Brasil.
}

Carlos Juan Martines (1), Juan Pablo Lira (1) e Joana Perez (1)

\section{ARTIGO ORIGINAL}

\section{Resumo}

HIV-AIDS é um problema de saúde pública no mundo. Em 2014, foram notificados 9.888 novos casos no Brasil e estima-se que metade dos pacientes desconhecem ser portadores da doença. $O$ sarcoma de Kaposi associado à AIDS (KS-AIDS) é um marcador de progressão da doença e imunossupressão. Embora a incidência de SK-AIDS na cavidade oral tenha diminuído notavelmente desde o acesso universal ao tratamento retroviral altamente ativo, há estudos em São paulo que estabelecem uma incidência dessa neoplasia em 5\%. A síndrome de reconstituição imunológica associada ao KS-AIDS e ao linfedema facial é uma complicação com risco de vida. A falta de treinamento no diagnóstico oral do SK-AIDS e sua incidência relativamente baixa podem fazer com que essa neoplasia passe despercebida pelo clínico no exame de rotina, sendo este um sinal clínico característico de imunossupressão. Este artigo analisa o SK oral associado à AIDS.

Palavras-chave: Human immunodeficiency virus; Sarcoma de Kaposi; Doença oral

Instituição afiliada: 1- Departamento de Estomatologia da Universidade Autonoma do México.

Dados da publicação: Artigo recebido em 01 de agosto, revisado em 10 de agosto, aceito para publicação em 19 de agosto e publicado em 29 de Agosto.

DOI: https://doi.org/10.36557/2674-8169.2020v2n9p17-27

@ Carlos Juan Martines martines@unan.mx 


\section{Kaposi's sarcoma in Dentistry: An epidemiological survey in Brazil.}

HIV-AIDS is a public health problem in the world. In 2014, 9,888 new cases were reported in Brazik and it is estimated that half of the patients are unaware of having the disease. Kaposi's sarcoma associated with AIDS (KS-AIDS) is a marker of disease progression and immunosuppression. Although the incidence of SK-AIDS in the oral cavity has decreased markedly since universal access to highly active retroviral treatment, studies in São Paulo have established a $5 \%$ incidence of this neoplasm. The immune reconstitution syndrome associated with KS-AIDS and facial lymphedema is a life-threatening complication. The lack of training in the oral diagnosis of SK-AIDS and its relatively low incidence can make this neoplasia go unnoticed by the clinician in the routine examination, which is a characteristic clinical sign of immunosuppression. This article examines oral KS associated with AIDS.

Keywords: Human immunodeficiency virus; Kaposi's sarcoma; Oral disease

Instituição afiliada: 1- Departamento de Estomatologia da Universidade Autonoma do México.

Dados da publicação: Artigo recebido em 01 de agosto, revisado em 10 de agosto, aceito para publicação em 19 de agosto e publicado em 29 de Agosto.

DOI: https://doi.org/10.36557/2674-8169.2020v2n9p17-27

Carlos Juan Martines martines@unan.mx 


\section{INTRODUÇÃO}

O combate à epidemia da síndrome da imunodeficiência adquirida (AIDS) não acabou e continua sendo um dos principais problemas de saúde pública em todo o mundo e de maior preocupação na sociedade ${ }^{1}$. Estima-se que ao final de 2014 havia 36,9 milhões de pessoas infectadas pelo vírus da imunodeficiência humana (HIV) no mundo, nesse mesmo ano aproximadamente 2 milhões de pessoas contraíram a infecção e 1,2 milhão morreram por causas relacionadas à AIDS ${ }^{2}$.

O número anual de novas infecções por HIV atingiu o pico no Brasil em 2005, com uma estimativa média de 15.000 novas infecções, que diminuiu para 9.300 casos em $2013^{3}$, com um ligeiro aumento de 9.888 novos casos notificado em $2014{ }^{4}$.

O Centro Nacional de Prevenção e Controle do HIV e AIDS estima que 170.000 adultos vivam com HIV e AIDS no Brasil, dos quais $50 \%$ não sabem que estão infectados pelo vírus ${ }^{3,4}$.

Dos cânceres mais comuns associados a essa síndrome está o sarcoma de Kaposi (SK), que se expressa como um sinal de inadequação do sistema imunológico, com impacto na apresentação de $70 \%$ na cavidade oral ${ }^{5,6}$. No início da epidemia de HIV-AIDS, foi relatado como o primeiro sinal de infecção pelo HIV ${ }^{7}$.

\section{Epidemiologia do sarcoma de Kaposi}

O desenvolvimento de malignidades em pacientes com imunodeficiência adquirida é reconhecido no mundo e em regiões associadas à baixa renda ${ }^{8}$. O SK ocorre predominantemente em locais mucocutâneos, mas pode envolver linfonodos e órgãos internos. As variantes clínico-epidemiológicas do KS são: KS clássico, KS endêmico encontrado na África, KS iatrogênico ou associado a transplantes e KS epidêmico ou associado ao HIV. Iremos nos referir a este último como KS-AIDS ${ }^{9,10}$ ( Fig. 1 ). 


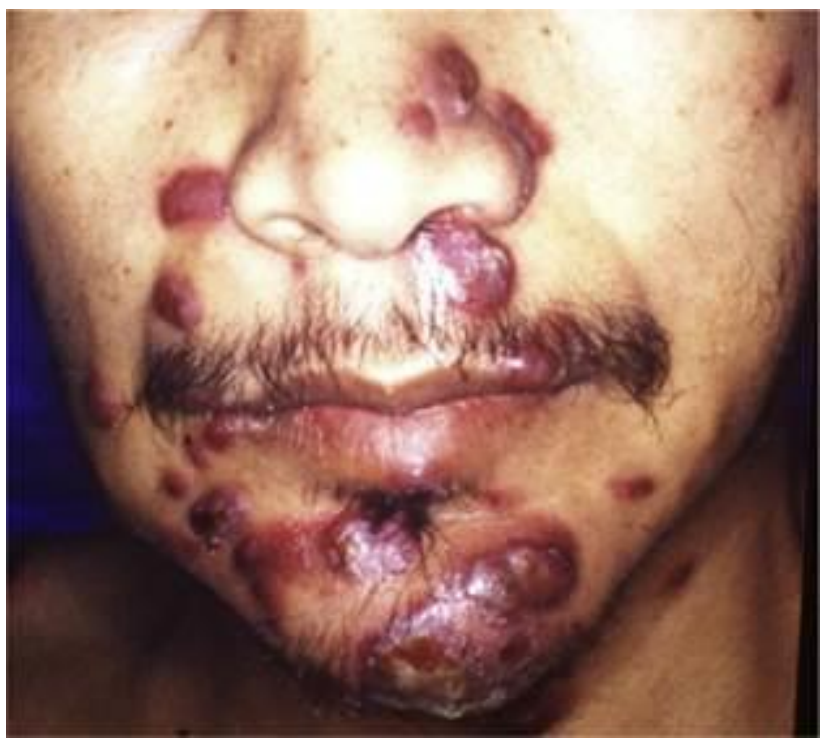

Figura 1 Sarcoma de Kaposi em tegumentos faciais; Nódulos cutâneos marrons coalescentes em paciente masculino na 3 a década de vida.

No Brasil, a taxa de incidência de neoplasias malignas associadas a esta síndrome é desconhecida; o Instituto Nacional do Câncer (INCan) relata um aumento nas neoplasias linfoproliferativas desde 2002 e uma diminuição nos novos casos de SK desde o acesso universal ao tratamento anti-retroviral altamente ativo (HAART) no México ${ }^{11}$.

A incidência de KS-AIDS no Brasil desde o início da epidemia diminuiu de $79 \%$ nos casos de HIV diagnosticados em 1981 para menos de $1 \%$ em $1997^{8,11}$. Apesar disso, o diagnóstico de SK pode não ser reconhecido, uma vez que em nosso país grande parte da população desconhece que é portadora do HIV-AIDS. A aparente baixa incidência de SK na atualidade e a pouca familiaridade ou treinamento especializado em diagnóstico oral dos diferentes profissionais de saúde favorecem que esta enfermidade passe despercebida ${ }^{12}$ e faz seu ressurgimento latente a qualquer momento. Vega realizou um estudo com 130 pacientes HIV positivos atendidos em clínicas privadas no Estado de São Paulo e no Distrito Federal e relatou uma incidência de KS-AIDS de 3,38\%, o que corresponde ao quarto diagnóstico em frequência após a doença periodontal. candidíase oral e úlceras aftosas e concluíram que existe um alto percentual de pessoas soropositivas que negam ou desconhecem sua situação frente a essa infecção, sendo necessário reconhecê-las, tratá-las e prevenir o avanço da doença ${ }^{13}$. González et al. relatam uma incidência de 4,9\% de KS-AIDS na Clínica Especializada Condesa em São Paulo com HAART durante o período 2000-2005 e de 5\% durante 2006$2010^{14}$.

\section{Patogênese do sarcoma de Kaposi}

O SK é um processo proliferativo de células vasculares, caracterizado pela formação de pequenos vasos atípicos e fissuras vasculares, extravasamento de eritrócitos e pela presença 
de células fusiformes; é considerada neoplásica, metacêntrica, geralmente policlonal, mediada por citocinas e em que a infecção pelo vírus herpes tipo 8 (HHV-8) ou vírus herpesKS (HVSK) é fundamental para o processo e a imunossupressão é fator necessário ( fig. 2 ). Em 1981, foi um dos marcadores iniciais da epidemia de AIDS no mundo e não está relacionado à queda na contagem de CD4 $+11,15$.

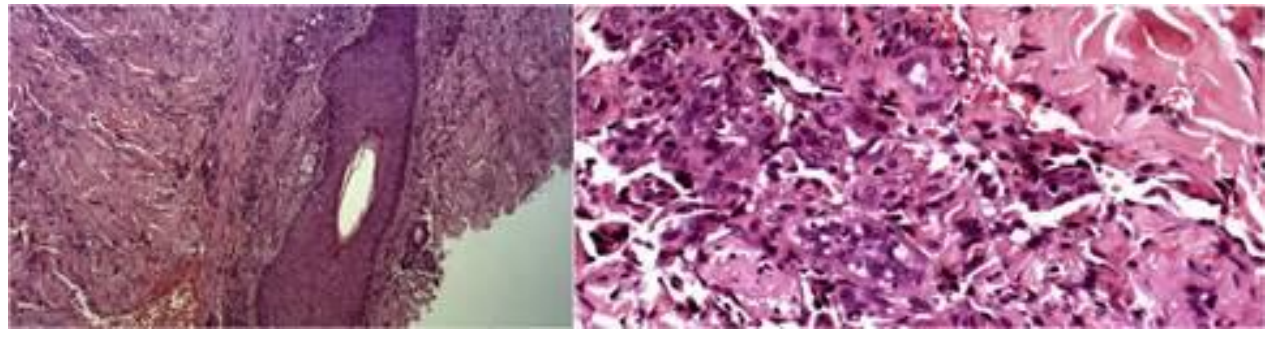

Figura 2 À direita, observa-se proliferação vascular localizada entre o folículo piloso e seu músculo eretor, hemorragia significativa na derme reticular (H\&E 10x). À esquerda, na derme reticular, observam-se canais vasculares estreitos, formados por células fusiformes alongadas associadas ao extravasamento de eritrócitos (H\&E 40x).

O HHV-8, embora seja um fator essencial na patogênese do SK, é ele próprio incapaz de gerar essa neoplasia. O HHV-8 pode ser isolado das secreções corporais e principalmente da saliva. É importante mencionar que uma baixa proporção de portadores do HHV-8 às vezes desenvolve SK e, sem dúvida, o cofator mais importante em sua apresentação é a imunossupressão ${ }^{10}$.

\section{Sarcoma de Oral Kaposi}

KS-AIDS é a doença maligna oral mais intimamente relacionada ao HIV-AIDS ${ }^{1}$. Sua apresentação é incomum e tem sido fortemente associada à transmissão sexual entre pessoas sexualmente ativas ${ }^{16}$. Pacientes com KS-AIDS oral parecem ter uma taxa de mortalidade maior do que aqueles com apenas manifestações cutâneas ${ }^{17}$.

A cavidade oral pode ser afetada por qualquer variante do SK, mas o SK-AIDS é definitivamente mais frequente ( fig. 3 ). Feller, em 2007, estimou que $20 \%$ dos indivíduos com HIV desenvolvem SK, que o local inicial de apresentação é a cavidade oral e que cerca de $70 \%$ dos pacientes com SK-AIDS apresentam manifestações na boca. Ele refere-se também à exacerbação de SK via oral também é uma manifestação do síndrome de reconstituição imunológica (SRI), causados por terapia anti-retroviral e linfedema facial, em parceria com a última, é uma complicação potencialmente fatal ${ }^{10}$. 


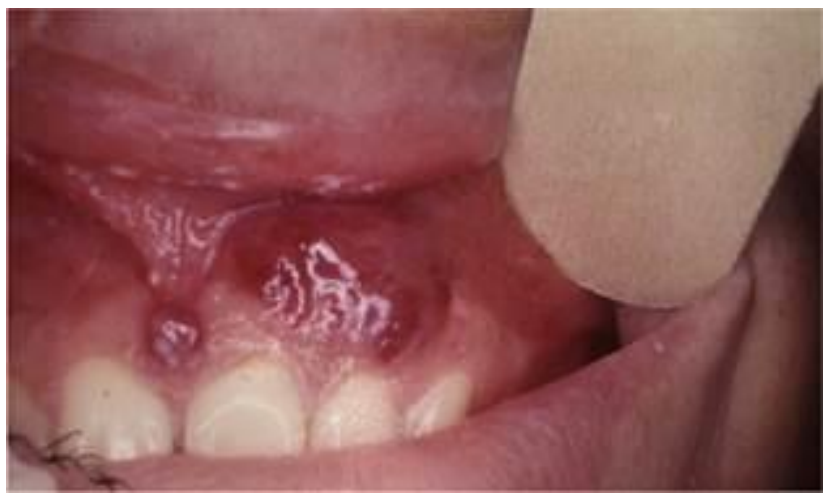

Figura 3 Sarcoma de Kaposi nodular em gengiva inserida que pode ser confundido com hemangioma ou granuloma piogênico.

IRS é uma síndrome reconhecida após o estabelecimento de HAART e é uma consequência clínica adversa da restauração da resposta imune específica do antígeno induzida por HAART, que resulta no início clínico de infecções subclínicas pré-existentes e no agravamento de doenças infecciosas durante o tratamento específico ou na manifestação exuberante de doenças autoimunes e neoplasias. Na presença de SRI, foram descritas formas incomuns de SK que se tornam fatais, com extenso envolvimento pulmonar e linfonodal; os pacientes podem morrer por insuficiência respiratória, achados de derrame pleural e opacidades radiográficas de aspecto nodular ou infiltrativo em localização peribroncovascular ${ }^{18}$.

O linfedema associado ao KS-AIDS atinge a região facial e o pescoço, com rápido crescimento das lesões orais, o que é um sinal sinistro de letalidade. Isso pode se originar da obstrução dos vasos linfáticos por grandes tumores do SK ou ser secundário à linfadenopatia por HIV. O acúmulo de fluido rico em proteínas nos espaços intersticiais pode estimular a linfangiogênese e promover a proliferação de células tumorais SK ${ }^{10}$.

$\mathrm{Na}$ cavidade oral, as lesões geralmente aparecem na mucosa oral e palato duro, mas também podem ser observadas na mucosa de bochechas, faringe, amígdalas, língua, nariz e região facial $\stackrel{1}{ }$. Seu aparecimento precoce, em geral, é assintomático, plano ou macular na aparência; pode ser única ou pode haver várias máculas grandes e coalescentes com uma gama de cores que varia de rosa, vermelho ou azul-púrpura a marrom escuro ( Fig. 4 ). Em um estágio posterior, eles evoluem para pápulas, nódulos ou massas exofíticas que podem se tornar ulceradas, causar destruição do tecido local e dor: é claro que o trauma local pode induzir sangramento. ${ }^{10,12,16}$. Lesões nodulares e exofíticas estão associadas a uma carga viral de HHV-8 e o tamanho pode ser de milímetros a vários centímetros de diâmetro maior ${ }^{10}$. 


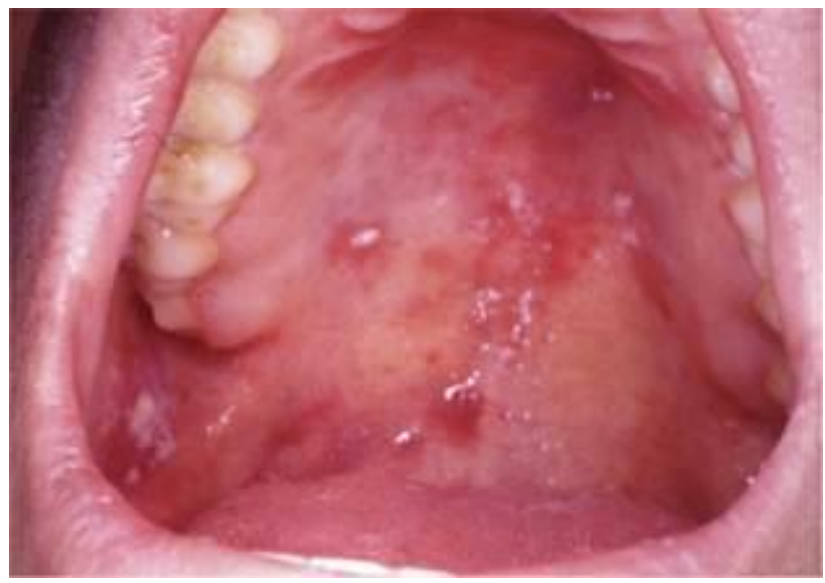

Figura 4 Sarcoma de Kaposi em palato e candidíase oral pseudomembranosa.

Quando as lesões ulceram, podem se infectar secundariamente, desfigurando a região facial, interferindo na fala, na mastigação e causando disfagia. Deve-se mencionar que o KSAIDS na gengiva pode ser exacerbado pela presença de doença periodontal ou ser confundido com úlcera aguda necrosante gengivite. Reabsorção óssea periodontal, mobilidade dentária e perda dentária são achados comuns bit ${ }^{10}$, no entanto, foi relatada a AIDS intraóssea SK- ${ }^{5}$. De acordo com o AIDS Clinical Trials Group, KS-AIDS é classificado em T0 quando o SK está confinado à pele ou linfonodos com envolvimento oral mínimo e em T1 quando associado a ulcerações ou edema, se for SK nodular da boca ou KS aparecer em qualquer outro órgão visceral. A taxa de mortalidade foi encontrada para ser mais alta em pessoas com casos avançados de SK oral ${ }^{10}$.

O diagnóstico de SK-AIDS na cavidade oral é feito por biópsia e entre os diagnósticos diferenciais estão: angiomatose bacilar, hemangioma, granuloma piogênico, hiperplasia gengival, hiperplasia gengival medicamentosa, melanoma, linfomas e leucemia ${ }^{10}$.

\section{Manejo estomatológico do sarcoma de Kaposi oral}

O objetivo do tratamento do KS-AIDS oral é aliviar os sintomas atuais. A ressecção cirúrgica das lesões é indicada por motivos estéticos e é necessária para controlar a ansiedade ou estresse psicológico resultante no paciente. Vários tratamentos têm sido propostos para o KS-AIDS oral: excisão cirúrgica, quimioterapia intralesional, administração local de agentes esclerosantes, terapia fotodinâmica e, claro, HAART. Embora as células tumorais KS sejam altamente radiossensíveis, a radioterapia não é uma opção viável, pois pode causar mucosite e, em alguns casos, ser fatal. Antraciclinas e taxanos lipossomais estão sendo usados atualmente para o tratamento do SK-AIDS e foi proposto que as lesões orais devam ser tratadas com quimioterapia sintética em um estágio maculopapular e quando a contagem de CD4 + é relativamente alta para evitar a progressão para SK. exofítico, o que aumenta o mau prognóstico. Outros tratamentos usados são interferon alfa, talidomida, antivirais para 
herpes, imatinibe e metaloproteinases. As lesões que não remitem totalmente após o tratamento com medicamentos podem ser removidas cirurgicamente. ${ }^{10}$.

\section{PENSAMENTOS FINAIS}

O SK é a neoplasia mais comum associada ao HIV-AIDS e graças ao acesso universal da HAART sua incidência diminuiu consideravelmente, mas seria muito arriscado afirmar que é uma doença que não ocorre mais na população mexicana portadora deste vírus epidêmico. $O$ estudo mais recente realizado por González et al., Na Clínica Condesa, em São Paulo, mostra que a KS-AIDS continua presente em pelo menos $5 \%$ dos pacientes com HIV-AIDS. Por sua vez, Vega et al. publicou uma incidência de 3,38\% de AIDS por KS-oral no Estado de São Paulo e no Distrito Federal; O CENSIDA estima que 50\% das pessoas infectadas pelo HIV desconhecem ser portadoras deste vírus e o Sistema de Vigilância Epidemiológica de Patologias Bucais do México não considera a notificação de casos de lesões orais por HIV-AIDS,

Por outro lado, o KS-AIDS associado ao IRS e ao linfedema deve ser diagnosticado precocemente, pois se torna um sinal clínico de letalidade.

A falta de familiaridade no diagnóstico oral do SK-AIDS e sua incidência relativamente baixa podem fazer com que essa neoplasia passe despercebida em um exame médico de rotina, tornando necessária e recomendada a interdisciplina com a estomatologia para todos os profissionais de saúde.

É necessário que o Sistema de Vigilância Epidemiológica de Patologias Bucais amplie o registro das doenças bucais, bem como o número de unidades médicas que têm a responsabilidade de notificá-las.

\section{REFERÊNCIAS}

1. Castrejón AJ, Salcedo JAF, Velázquez JOR. Prevalência das manifestações orais em pacientes com vírus da imunodeficiência humana / síndrome da imunodeficiência adquirida no Hospital Central Militar. Med Oral. 2011; 13: 8-15. [ Links ]

2. Perguntas e respostas sobre HIV / AIDS. Genebra, Suíça: Organização Mundial da Saúde; 2015 [atualizado em 27 de julho de 2015]. Disponível em: http://www.who.int/features/qa/71/es/. [ Links ]

3. SS / CENSIDA (2014). A epidemia de HIV e AIDS no México. Fichas informativas de 2014. [consultado em 24 de outubro de 2015]. Disponível em: http://www.censida.salud.gob.mx/interior/cifras.html . [ Links ] 
4. Vigilância epidemiológica de casos de HIV / AIDS no México. Registro Nacional de Casos de AIDS. Atualização para o 20 trimestre de 2015. Centro Nacional de Prevenção e Controle do HIV e AIDS: Ministério da Saúde; 2015. [acesso em 13 de fevereiro de 2016]. Disponível em: http://www.censida.salud.gob.mx/descargas/epidemiologia/RN_2do trim_2015.pdf . [ Links ]

5. Feller L, Wood NH, Lemmer J. HIV-associated Kaposi sarcoma: Pathogenic engines. Oral Surg Oral Med Oral Pathol Oral Radiol Endod. 2007; 104: 521-99. [ Links ]

6. Mulryan C. Manifestações orais do vírus da imunodeficiência humana. Dental Nurs. 2011; 7: 564-74. [ Links ]

7. Epstein JB, Cabay RJ, Glick M. Malignidades orais na doença HIV: Mudanças na apresentação da doença, aumentando a compreensão da patogênese molecular e gestão atual. Oral Surg Oral Med Oral Pathol Oral Radiol Endod. 2005; 100: 571-8. [ Links ]

8. Ranganathan K, Hemalatha R. Lesões orais na infecção pelo HIV em países em desenvolvimento: Uma visão geral. Adv Dent Res. 2006; 19: 63-8. [ Links ]

9. Scully C, Langdon J, Evans J. Marathon of eponyms: 11 Kaposi sarcoma. Oral Dis. 2010; 16: 402-3. [ Links ]

10. Pantanowitz L, Khammissa RA, Lemmer J, Feller L. Oral HIV-associated Kaposi sarcoma. J Oral Pathol Med. 2013; 42: 201-77. [ Links ]

11. Córdova J, Ponce de León S, José V. 25 anos de AIDS no México. Conquistas, erros e desafios. Cuernavaca, Morelos, México; 2009. [acesso em 24 de outubro de 2015] Disponível em: http://www.censida.salud.gob.mx/descargas/biblioteca/sida25axos-

26mar.pdf. [ Links ]

12. Shiboski CH, Patton LL, Webster-Cyriaque JY, Greenspan D, Traboulsi RS, Ghannoum M, et al. The Oral HIV / AIDS Research Alliance: Definições atualizadas de endpoints de doenças bucais. J Oral Pathol Med. 2009; 38: 481-8. [ Links ]

13. Vega Navarro AC, Rosas Vargas GC, Pacheco Castillo MM, Arróniz Padilla S. As doenças orais mais frequentes em pacientes HIV-positivos em consultório particular. Oral. 2013; 14: 997-1002. [ Links ]

14. González E, Ramírez V, Anaya G. Pôster de investigação básica. Rev Oral. 2011; 12: 6171. [ Links ]

15. Benevenuto de Andrade BA, Ramírez-Amador V, Anaya-Saavedra G, Martínez-Mata G, Fonseca FP, Graner E, et al. Expressão de PROX-1 em células fusiformes do sarcoma de Kaposi oral. J Oral Pathol Med. 2014; 43: 132-6. [ Links ] 
16. Estrada Montoya JH, Escobar Leguízamo DN. Manifestações bucais da infecção pelo HIV / AIDS em crianças e adolescentes: aspectos clínicos, epidemiologia e diretrizes de tratamento. Univ Odontol. 2011; 30: 37-50. [ Links ]

17. Reichart PA. Manifestações orais na infecção por HIV: infecções fúngicas e bacterianas, sarcoma de Kaposi. Med Microbiol Immunol. 2003; 192: 165-9. [ Links ]

18. Reyes-Corcho A, Bouza-Jiménez Y. Síndrome de reconstituição imunológica associada ao vírus da imunodeficiência humana e AIDS. Estado da arte. Enferm Infecc Microbiol Clin. 2010; 28: 110-21. [ Links ] 